\title{
Symbolic models approximating possibly unstable time-delay systems with application to the artificial pancreas
}

\author{
Giordano Pola, Alessandro Borri, Pierdomenico Pepe, Pasquale Palumbo and Maria D. Di Benedetto
}

\begin{abstract}
Symbolic models are becoming more and more popular in the research community working on hybrid systems because they provide a systematic approach to enforce logic specifications on purely continuous or hybrid systems while respecting the constraints at the hardware/software implementation level. This paper contributes to this research line and proposes symbolic models approximating possibly unstable timedelay systems with quantized measurements of the outputs. Application of the proposed results to the glucose control problems for the Artificial Pancreas is discussed in the paper.
\end{abstract}

\section{INTRODUCTION}

Symbolic models are abstract descriptions of continuous systems where states correspond to aggregates of continuous states [1]. During the years, this paradigm has been shown to be able to provide a systematic approach to the formal verification and the control design of complex heterogeneous processes, as e.g. cyber-physical systems; see e.g. [1], [2], [3] and the references therein. With the present paper we contribute to this research line and propose symbolic models approximating possibly unstable time-delay systems with quantized measurements of outputs in the sense of alternating approximate simulation. For doing so, we first extend the notion of incremental forward completeness introduced in [4] for nonlinear systems and provide Lyapunov-Krasovskii like inequalities to check it. Then, as done in [5], [6] for incrementally stable time-delay systems without outputs, we use first order spline analysis, e.g. [7], for effectively constructing symbolic models. Output variables are handled by extending symbolic models proposed in [8] for nonlinear systems. Apart from the theoretical interest per se, a motivating reason for this research resides in setting an appropriate framework where to study glucose control problems for the Artificial Pancreas (AP). AP refers to the integration of cutting edge design of insulin infusion therapies with stateof-the-art technological devices involved in insulin administration actuators and continuous glucose measurements. Most of the available AP systems are thought for Type 1 Diabetes Mellitus (T1DM) patients (e.g. [9], [10], [11], [12], [13], [14]), i.e. patients who suffer for a complete lacking of the endogenous pancreatic insulin delivery, therefore requiring an exogenous insulin administration throughout their life. In

G. Pola, P. Pepe and M.D. Di Benedetto are with the Department of Information Engineering, Computer Science and Mathematics, Center of Excellence DEWS, University of L'Aquila, 67100 L'Aquila, Italy, \{giordano.pola,pierdomenico.pepe,mariadomenica.dibenedetto\}@univaq.it. A. Borri and P. Palumbo are with the Istituto di Analisi dei Sistemi ed Informatica "A. Ruberti", Consiglio Nazionale delle Ricerche (IASI-CNR), 00185 Rome, Italy, \{alessandro.borri,pasquale.palumbo\}@ iasi.cnr.it.

This work has been partially supported by the Center of Excellence for Research DEWS, University of L'Aquila, Italy. this work we use symbolic models to design model-based, closed-loop glucose control therapies for Type 2 Diabetes Mellitus (T2DM) patients. T2DM involves the great majority of diabetic people (about 90\%), thus resulting in a large impact on National Health Systems, since without control, T2DM eventually facilitates the occurrence of a wide range of serious total-body diabetic complications. The chosen model-based approach exploits the mathematical model of the glucose-insulin system to design the control law. To this end a time-delay system, recently exploited for the AP in a T2DM framework, is considered (see [15], [16] for the model references and [17], [18] for the model-based AP application). Indeed, time-delay systems are known to best represent the endogenous pancreatic insulin delivery rate, therefore they have to be preferred to ODE models when dealing with T2DM [19], [20]. Glucose control problems consist in regulating the level of plasma glucose concentrations by means of exogeneous insulin infusions. We solve this control problem by using algorithms based on supervisory control [21], see also [22], on the symbolic models we propose. The main advantages of this approach with respect to the ones existing in the current literature are:

- it allows considering input signals that are delayed, modelling e.g. measurements/actuators processing or physiological compartments, and measurements and inputs that are quantized, as it is the case in the digital devices actually in use for glucose control;

- it does not impose (possibly small) upper bounds on the sampling interval, thus making our results implementable on low-cost devices.

We show a preliminary validation of the approach in some in-silico simulations, involving a population of virtual T2DM patients, with parameters perturbed with respect to the nominal values used in the symbolic model. The method shows how the controller is able to drive the individual glycemia towards healthier values, also preventing dangerous episodes of hypoglycemia.

This paper is organized as follows. Section II recalls notation. Section III introduces time-delay systems and the notion of incremental forward completeness. Section IV recalls notions on systems and approximate relations. Section $\mathrm{V}$ presents symbolic models that are then applied in Section VI to glucose control. Section VII offers some concluding remarks.

\section{Notation And PReliminary Definitions}

The symbols $\mathbb{N}_{0}, \mathbb{Z}, \mathbb{R}, \mathbb{R}^{+}$and $\mathbb{R}_{0}^{+}$denote the set of nonnegative integer, integer, real, positive real, and nonneg- 
ative real numbers, respectively. Given $a, b \in \mathbb{Z}$, we denote $[a ; b]=[a, b] \cap \mathbb{Z}$. Given a set $X$, the symbol $2^{X}$ denotes the power set of $X$. Given a pair of sets $X$ and $Y$ and a relation $\mathcal{R} \subseteq X \times Y$, the symbol $\mathcal{R}^{-1}$ denotes the inverse relation of $\mathcal{R}$, i.e. $\mathcal{R}^{-1}=\{(y, x) \in Y \times X:(x, y) \in \mathcal{R}\}$. Given $X^{\prime} \subseteq X$ and $Y^{\prime} \subseteq Y$, we denote $\mathcal{R}\left(X^{\prime}\right)=\{y \in$ $Y \mid \exists x \in X^{\prime}$ s.t. $\left.(x, y) \in \mathcal{R}\right\}$ and $\mathcal{R}^{-1}\left(Y^{\prime}\right)=\{x \in X \mid \exists y \in$ $Y^{\prime}$ s.t. $\left.(x, y) \in \mathcal{R}\right\}$. Given a function $f: X \rightarrow Y$, the symbol $f^{-1}: Y \rightarrow 2^{X}$ denotes the inverse map of $f$, i.e., $f^{-1}(y)=\{x \in X: y=f(x)\}$ for all $y$ in the codomain of $f$. Given $f$ and $X^{\prime} \subseteq X$ the symbol $f\left(X^{\prime}\right)$ denotes the image of $X^{\prime}$ through $f$, i.e. $f\left(X^{\prime}\right)=\{y \in$ $Y \mid \exists x \in X^{\prime}$ s.t. $\left.y=f(x)\right\}$. Similarly, given $Y^{\prime} \subseteq Y$ the symbol $f^{-1}\left(Y^{\prime}\right)$ denotes the inverse image of $Y^{\prime}$ through $f$, i.e. $f^{-1}\left(Y^{\prime}\right)=\left\{x \in X \mid f(x) \in Y^{\prime}\right\}$. Given $X^{\prime} \subset X$ the symbol $\left.f\right|_{X^{\prime}}$ denotes the restriction of $f$ to $X^{\prime}$ that is $\left.f\right|_{X^{\prime}}: X^{\prime} \rightarrow Y$ such that $\left.f\right|_{X^{\prime}}\left(x^{\prime}\right)=f\left(x^{\prime}\right)$ for all $x^{\prime} \in X^{\prime}$. A continuous function $\gamma: \mathbb{R}_{0}^{+} \rightarrow \mathbb{R}_{0}^{+}$is said to belong to class $\mathcal{K}$ if it is strictly increasing and $\gamma(0)=0$; function $\gamma$ is said to belong to class $\mathcal{K}_{\infty}$ if $\gamma \in \mathcal{K}$ and $\gamma(r) \rightarrow \infty$ as $r \rightarrow \infty$. Symbol $I_{r}$ denotes the identity matrix in $\mathbb{R}^{r}$. Given a vector $x \in \mathbb{R}^{n}$ we denote by $x(i)$ the $i$-th element of $x$ and by $\|x\|$ the infinity norm of $x$. Given $a \in \mathbb{R}$ and $X \subseteq \mathbb{R}^{n}$, the symbol $a X$ denotes the set $\left\{y \in \mathbb{R}^{n} \mid \exists x \in X\right.$ s.t. $\left.y=a x\right\}$. Given $\theta \in \mathbb{R}^{+}$and $x \in \mathbb{R}^{n}$, we denote $\mathcal{B}_{[-\theta, \theta[}^{n}(x)=\{y \in$ $\mathbb{R}^{n} \mid y(i) \in[-\theta+x(i), \theta+x(i)[, i \in[1 ; n]\}$. Note that for any $\theta \in \mathbb{R}^{+}$, the collection of $\mathcal{B}_{[-\theta, \theta]}^{n}(x)$ with $x$ ranging in $2 \theta \mathbb{Z}^{n}$ is a partition of $\mathbb{R}^{n}$. Given a positive $n \in \mathbb{N}_{0}$ and quantization parameter $\theta \in \mathbb{R}^{+}$, the quantizer in $\mathbb{R}^{n}$ with accuracy $\theta$ is a function $[\cdot]_{\theta}^{n}: \mathbb{R}^{n} \rightarrow 2 \theta \mathbb{Z}^{n}$ associating to any $x \in \mathbb{R}^{n}$ the unique vector $[x]_{\theta}^{n} \in 2 \theta \mathbb{Z}^{n}$ such that $x \in \mathcal{B}_{[-\theta, \theta[}^{n}\left([x]_{\theta}^{n}\right)$. Definition of $[\cdot]_{\theta}^{n}$ naturally extends to sets $X \subseteq \mathbb{R}^{n}$ when $[X]_{\theta}^{n}$ is interpreted as the image of $X$ through function $[\cdot]_{\theta}^{n}$. Given a measurable and essentally bounded function $f: \mathbb{R}_{0}^{+} \rightarrow \mathbb{R}^{n}$, the (essential) supremum norm of $f$ is denoted by $\|f\|_{\infty}$; we recall that $\|f\|_{\infty}:=($ ess $) \sup \{\|f(t)\|$, $t \geq 0\}$. For a given time $\tau \in \mathbb{R}^{+}$, define $f_{\tau}$ so that $f_{\tau}(t)=f(t)$, for any $t \in[0, \tau[$, and $f(t)=0$ elsewhere; $f$ is said to be locally essentially bounded if for any $\tau \in \mathbb{R}^{+}$, $f_{\tau}$ is essentially bounded. Given $k, n \in \mathbb{N}$ with $n \geq 1$ and $I=[a, b] \subseteq \mathbb{R}, a, b \in \mathbb{R}, a<b$ let $C^{k}\left(I ; \mathbb{R}^{n}\right)$ be the space of functions $f: I \rightarrow \mathbb{R}^{n}$ that are continuously differentiable $k$ times. Given $k \geq 1$, let $P C^{k}\left(I ; \mathbb{R}^{n}\right)$ be the space of $C^{k-1}\left(I ; \mathbb{R}^{n}\right)$ functions $f: I \rightarrow \mathbb{R}^{n}$ whose $k$-th derivative exists except in a finite number of reals, and it is bounded, i.e. there exist $\gamma_{0}, \gamma_{1}, \ldots, \gamma_{s} \in \mathbb{R}^{+}$with $a=\gamma_{0}<\gamma_{1}<\ldots<\gamma_{s}=b$ so that $D^{k} f$ is defined on each open interval $\left(\gamma_{i}, \gamma_{i+1}\right), i=0,1, \ldots, s-1$ and $\max _{i=0,1, \ldots, s-1} \sup _{t \in\left(\gamma_{i}, \gamma_{i+1}\right)}\left\|D^{k} f(t)\right\|_{\infty}<\infty$. For any continuous function $x(s)$, defined on $-\Delta \leq s<a, a>0$, and any fixed $t, 0 \leq t<a$, the standard symbol $x_{t}$ will denote the element of $C^{0}\left([-\Delta, 0] ; \mathbb{R}^{n}\right)$ defined by $x_{t}(\theta)=$ $x(t+\theta),-\Delta \leq \theta \leq 0$.

\section{TIME-DElay Systems}

In this paper we consider the following nonlinear timedelay system with quantized measurements of the output:

$$
\begin{cases}\dot{x}(t)=f\left(x_{t}, u(t-r)\right), & t \in \mathbb{R}^{+}, \text {a.e. } \\
x_{0} \in \mathcal{X}_{0}, & \\
y(t)=[z(t)]_{\lambda}^{p}, \text { with } z(t)=\left[\begin{array}{ll}
I_{p} & 0
\end{array}\right] x(t), \quad t \in \mathbb{R}_{0}^{+},\end{cases}
$$

where: $\Delta \in \mathbb{R}_{0}^{+}$is the maximum involved state delay; $r \in \mathbb{R}_{0}^{+}$is the constant input delay; $x(t) \in X \subseteq \mathbb{R}^{n}$, $x_{t} \in \mathcal{X}=C^{0}([-\Delta, 0] ; X)$ is the state at time $t \in \mathbb{R}_{0}^{+}$; $u(t) \in U \subseteq \mathbb{R}^{m}$ is the control input at time $t \in[-r,+\infty[$ $y(t) \in \mathbb{R}^{p}$ is the output at time $t \in \mathbb{R}_{0}^{+}$, obtained as the quantization with accuracy $\lambda \in \mathbb{R}^{+}$of $\left[\begin{array}{ll}I_{p} & 0\end{array}\right] x(t)$, where $x(t)$ is the last value of state $x_{t} ; f$ is a functional from $\mathcal{X} \times U$ to $\mathbb{R}^{n} ; \mathcal{X}_{0} \subseteq \mathcal{X}$ is the set of initial states. Control inputs are assumed to be piecewise-constant and take value in $U$ which is assumed to be a finite set. More formally, let $\mathcal{U}_{\tau}$ be the collection of functions from $[-r,-r+\tau$ [ to $U$ with $u(t)=u(-r)$ for any $t \in[-r,-r+\tau[$, where $\tau \in \mathbb{R}^{+}$can be thought of as a sampling time parameter in the digital controller. The class of control input functions we consider, denoted $\mathcal{U}$, is then obtained as the concatenation of control input functions belonging to $\mathcal{U}_{\tau}$. From (1), we are assuming that the output variables of the system are the quantization of a selection of the state variables. The general case of nonlinear output functions can be considered at the expense of a heavier notation, as done in [23]. We suppose that $f$ is Lipschitz on bounded sets, i.e. for every bounded set $K \subset \mathcal{X} \times U$, there exists a constant $\kappa \in \mathbb{R}^{+}$such that $\left\|f\left(x_{1}, u_{1}\right)-f\left(x_{2}, u_{2}\right)\right\| \leq \kappa\left(\left\|x_{1}-x_{2}\right\|_{\infty}+\left\|u_{1}-u_{2}\right\|\right)$, for all $\left(x_{1}, u_{1}\right),\left(x_{2}, u_{2}\right) \in K$. We assume that there exists a constant function (state) $\phi_{e} \in \mathcal{X}$ such that $f\left(\phi_{e}, 0\right)=0$. Assumptions on $f$ ensure existence and uniqueness of the solution of the differential equation in (1), in a maximal time interval $[0, b), 0<b \leq+\infty$. In the following $x\left(t, \xi_{0}, u\right)$ and $x_{t}\left(\xi_{0}, u\right)$ will denote the solution in $X$ and respectively in $\mathcal{X}$, of the time-delay system with initial condition $\xi_{0} \in \mathcal{X}_{0}$ and input $u \in \mathcal{U}$, at time $t$. A time-delay system is said to be forward complete if every solution is defined on $[0,+\infty[$. In the further developments, we refer to a time-delay system as in (1) by means of the tuple:

$$
\Sigma=\left(X, \mathcal{X}, \mathcal{X}_{0}, U, \mathcal{U}, \mathbb{R}^{p}, f\right),
$$

where each entity has been defined before. We now introduce some assumptions that are made to derive our results. The following definition has been obtained as a natural generalization of the one given for nonlinear systems in [4] to time-delay systems.

Definition 1: A time-delay system

$$
\Sigma=\left(\mathbb{R}^{n}, \mathcal{X}, \mathcal{X}_{0}, U, \mathcal{U}, \mathbb{R}^{p}, f\right),
$$

is incrementally forward complete $(\delta-\mathrm{FC})$ if it is forward complete and there exists a continuous function $\beta$ such that for every $s \in \mathbb{R}_{0}^{+}, \beta(\cdot, s)$ belongs to class $\mathcal{K}_{\infty}$, and for any time $t \in \mathbb{R}_{0}^{+}$, any initial conditions $\xi_{1}, \xi_{2} \in \mathcal{X}$ and any 
$u \in \mathcal{U}$, the following inequality holds:

$$
\left\|x_{t}\left(\xi_{1}, u\right)-x_{t}\left(\xi_{2}, u\right)\right\|_{\infty} \leq \beta\left(\left\|\xi_{1}-\xi_{2}\right\|_{\infty}, t\right) .
$$

Note that time-delay system in (3) differs from the one in (2), (only) because the set of states in (3) is $\mathcal{X}=$ $C^{0}\left([-\Delta, 0] ; \mathbb{R}^{n}\right)$ while $\mathcal{X}=C^{0}([-\Delta, 0] ; X)$ in (2). We consider here $\Sigma$ as in (3) because $\delta$-FC is a notion that is global with respect to the state. In the sequel, if not explicitly referring to (3), time-delay system $\Sigma$ is as in (2). We now show that the $\delta$-FC property can be checked by resorting to Lyapunov-Krasovskii functional type inequalities.

Definition 2: A locally Lipschitz functional $V: \mathcal{X} \times \mathcal{X} \rightarrow$ $\mathbb{R}_{0}^{+}$is said to be a $\delta$-FC Lyapunov-Krasovskii functional for $\Sigma$ in (3) if there exist functions $\alpha_{1}, \alpha_{2}$ of class $\mathcal{K}_{\infty}, \sigma$ of class $\mathcal{K}$ and $p \in \mathbb{R}$ such that, for any $\phi, \psi \in \mathcal{X}$, for any $u \in \mathcal{U}$, the following inequalities hold

i) $\alpha_{1}(\|\phi(0)-\psi(0)\|) \leq V(\phi, \psi) \leq \alpha_{2}\left(\|\phi-\psi\|_{\infty}\right)$,

ii) $D^{+} V((\phi, u),(\psi, u)) \leq p V(\phi, \psi)$,

iii) $D^{+} V\left((\phi, u),\left(\phi_{e}, 0\right)\right) \leq p V\left(\phi, \phi_{e}\right)+\sigma(\|u\|)$

where

$$
\begin{gathered}
D^{+} V((\phi, u),(\psi, u))=\limsup _{h \rightarrow 0^{+}} \frac{V\left(\phi_{h, u}, \psi_{h, u}\right)-V(\phi, \psi)}{h}, \\
D^{+} V\left((\phi, u),\left(\phi_{e}, 0\right)\right)=\limsup _{h \rightarrow 0^{+}} \frac{V\left(\phi_{h, u}, \phi_{e}\right)-V\left(\phi, \phi_{e}\right)}{h}, \\
\phi_{h, u}(s)=\left\{\begin{array}{cc}
\phi(s+h), & s \in[-\Delta,-h) \\
\phi(0)+(s+h) f(\phi, u), & s \in[-h, 0], \\
\psi(s+h), & s \in[-\Delta,-h)
\end{array}\right. \\
\psi_{h, u}(s)=\left\{\begin{array}{cc}
(s+h) f(\psi, u), & s \in[-h, 0] \\
\psi(0)+(s+h) f
\end{array}\right.
\end{gathered}
$$

Theorem 1: If there exists a $\delta$-FC Lyapunov-Krasovskii functional for $\Sigma$ in (3), then $\Sigma$ is $\delta$-FC, with, for $s, t \in \mathbb{R}_{0}^{+}$,

$$
\beta(s, t)=\alpha_{1}^{-1}\left(e^{p(t+\Delta(h(p)-1))} \alpha_{2}(s)\right)
$$

where $h: \mathbb{R} \rightarrow\{0,1\}$ denotes the Heaviside function ${ }^{1}$.

When it is known a priori that all the solutions belong to a given compact set in $\mathbb{R}^{n}$, then the $\delta$-FC property can be characterized by conditions on Lyapunov-Krasovskii functionals suitably given on related bounded sets of $C^{0}\left([-\Delta, 0] ; \mathbb{R}^{n}\right)$.

\section{SYSTEMS AND APPROXIMATE RELATIONS}

In this paper we consider the notion of system as a unifying paradigm to describe time-delay systems as well as logic systems modeling controllers and specifications.

Definition 3: [1] A system is a tuple $S=$ $\left(X, X_{0}, U, \longrightarrow, Y, H\right)$ consisting of a set of states $X$, a set of initial states $X_{0} \subseteq X$, a set of inputs $U$, a transition relation $\longrightarrow \subseteq X \times U \times X$, a set of outputs $Y$ and an output function $H: X \rightarrow Y$.

In the sequel, a transition $\left(x, u, x^{\prime}\right) \in \longrightarrow$ of $S$ is denoted by $x \stackrel{u}{\longrightarrow} x^{\prime}$. For later purposes, given $x \in X$ define $U(x)$ as the collection of $u \in U$ for which there exists a transition

\footnotetext{
${ }^{1}$ We recall that $h(\cdot)$ is defined by $h(p)=1$, if $p \geq 0$, and $h(p)=0$, otherwise.
}

$x \stackrel{u}{\longrightarrow} x^{\prime}$ for some $x^{\prime} \in X$. The evolution of systems is captured by the notion of state run. Given a sequence $x_{0} \stackrel{u_{0}}{\longrightarrow} x_{1} \stackrel{u_{1}}{\longrightarrow} \ldots \stackrel{u_{l-1}}{\longrightarrow} x_{l}$ of transitions of $S$, with $x_{0} \in X_{0}$, the sequence $r_{X}: x_{0} x_{1} \ldots x_{l}$ is called a state run of $S$. System $S$ is said to be: countable, if $X$ and $U$ are countable sets; symbolic, if $X$ and $U$ are finite sets; metric, if $X$ is equipped with a metric $\mathbf{d}: X \times X \rightarrow \mathbb{R}_{0}^{+}$. In order to provide symbolic approximations of the time-delay system $\Sigma$, we need to recall the following notions.

Definition 4: [24] Consider a pair of metric systems

$$
S_{i}=\left(X_{i}, X_{0, i}, U_{i}, \underset{i}{\longrightarrow}, Y_{i}, H_{i}\right), i=1,2,
$$

with $X_{1}, X_{2} \subseteq X$ for some set $X$ equipped with metric d, and let $\eta \in \mathbb{R}^{+}$be a given accuracy. Consider a relation $\mathcal{R} \subseteq X_{1} \times X_{2}$, satisfying the following conditions:

(i) $\forall x_{1} \in X_{0,1} \exists x_{2} \in X_{0,2}$ such that $\left(x_{1}, x_{2}\right) \in \mathcal{R}$;

(ii) $\forall\left(x_{1}, x_{2}\right) \in \mathcal{R}, \mathbf{d}\left(x_{1}, x_{2}\right) \leq \eta$.

Relation $\mathcal{R}$ is a strong $\eta$-approximate (strong $\eta \mathrm{A}$ ) simulation relation from $S_{1}$ to $S_{2}$ if it enjoys conditions (i), (ii) and the following one:

(iii) $\forall\left(x_{1}, x_{2}\right) \in \mathcal{R}$ if $x_{1} \underset{1}{\stackrel{u_{1}}{\longrightarrow}} x_{1}^{\prime}$ then $\exists x_{2} \underset{2}{\stackrel{u_{2}}{\longrightarrow}} x_{2}^{\prime}$ with $u_{2}=u_{1}$ such that $\left(x_{1}^{\prime}, x_{2}^{\prime}\right) \in \mathcal{R}$.

System $S_{1}$ is strongly $\eta$-simulated by $S_{2}$, denoted $S_{1} \preceq_{\eta}^{s} S_{2}$, if there exists a strong $\eta$-approximate simulation relation from $S_{1}$ to $S_{2}$. Relation $\mathcal{R}$ is a strong alternating $\eta$ approximate (strong $A \eta A$ ) simulation relation from $S_{1}$ to $S_{2}$ if it enjoys conditions (i), (ii) and the following one: (iii') $\forall\left(x_{1}, x_{2}\right) \in \mathcal{R} \forall u_{1} \in U_{1}\left(x_{1}\right), u_{2}=u_{1} \in U_{2}\left(x_{2}\right)$ and $\forall x_{2} \underset{2}{\stackrel{u_{2}}{\longrightarrow}} x_{2}^{\prime} \exists x_{1} \stackrel{u_{1}}{\longrightarrow} x_{1}^{\prime}$ such that $\left(x_{1}^{\prime}, x_{2}^{\prime}\right) \in \mathcal{R}$.

System $S_{1}$ is strongly alternatingly $\eta$-simulated by $S_{2}$, denoted $S_{1} \preceq_{\eta}^{s, \text { alt }} S_{2}$, if there exists a strong $A \eta A$ simulation relation from $S_{1}$ to $S_{2}$.

\section{Approximate Symbolic Models}

In this section we propose symbolic models approximating time-delay systems. Results here reported generalize [5] from incrementally stable time-delay systems without outputs to $\delta$-FC time-delay systems with outputs. Given the time-delay system $\Sigma$ define the system

$$
S(\Sigma)=\left(X, X_{0}, U, \longrightarrow, Y, H\right),
$$

where $X=\mathcal{X}, X_{0}=\mathcal{X}_{0}, U=\left\{u \in \mathcal{U}_{\tau} \mid x_{\tau}(x, u)\right.$ is defined for all $x \in \mathcal{X}\}, x \stackrel{u}{\longrightarrow} x^{\prime}$ if $x_{\tau}(x, u)=x^{\prime}, Y=\mathbb{R}^{p}$, and $H(x)=[z]_{\lambda}^{p}$, with $z=\left[\begin{array}{ll}I_{p} & 0\end{array}\right] x(\tau)$, for all $x_{\tau} \in X$. System $S(\Sigma)$ captures the evolution of $\Sigma$ only at times $t=$ $k \tau$ with $k \in \mathbb{N}_{0}$. In this sense, $S(\Sigma)$ can be viewed as a time discretization of $\Sigma$. By definition of $X$, system $S(\Sigma)$ is metric. In the sequel we use the metric $\mathbf{d}\left(x, x^{\prime}\right)=\left\|x-x^{\prime}\right\|_{\infty}$, $x, x^{\prime} \in X$. Note that $S(\Sigma)$ is not symbolic, because the set of states $X$ is a functional space. The construction of symbolic models for time-delay systems relies upon approximations of the set of reachable states. Let $R_{\tau}(\Sigma) \subseteq \mathcal{X}$ be the set of reachable states of $\Sigma$ at times $t=0, \tau, \ldots, k \tau, \ldots$, i.e. the collection of all states $x \in \mathcal{X}$ for which there exist $k \in \mathbb{N}_{0}$ 
and a control input $u \in \mathcal{U}$ such that $x=x_{k \tau}\left(\xi_{0}, u\right)$, for some $\xi_{0} \in \mathcal{X}_{0}$. The set $R_{\tau}(\Sigma)$, corresponding to ${ }^{2} X$ in $S(\Sigma)$ is a functional space and therefore needs to be approximated. To this purpose, we use the following definition:

Definition 5: [5] Consider a functional space $\mathcal{Z} \subseteq$ $C^{0}(I ; Z)$ with $Z \subseteq \mathbb{R}^{n}, I=[a, b], a, b \in \mathbb{R}, a<b$. A map $\mathcal{A}: \mathbb{R}^{+} \rightarrow 2^{C^{0}(I ; Z)}$ is a countable approximation of $\mathcal{Z}$ if for any desired accuracy $\lambda \in \mathbb{R}^{+}$:

(i) $\mathcal{A}(\lambda)$ is a countable set;

(ii) for any $z \in \mathcal{Z}$ there exists $z^{\prime} \in \mathcal{A}(\lambda)$ such that

$$
\left\|z-z^{\prime}\right\|_{\infty} \leq \lambda
$$

(iii) for any $z^{\prime} \in \mathcal{A}(\lambda)$ there exists $z \in \mathcal{Z}$ s.t. (9) holds.

When condition i) above is replaced by:

(i') $\mathcal{A}(\lambda)$ is a finite set,

map $\mathcal{A}$ is said to be a symbolic approximation of $\mathcal{Z}$.

We first assume the existence of a countable approximation $\mathcal{A}_{\mathcal{X}}$ of $R_{\tau}(\Sigma)$. Map $\mathcal{A}_{\mathcal{X}}$ naturally induces a countable approximation $\mathcal{A}_{\mathcal{X}_{0}}$ of $\mathcal{X}_{0}$ because $\mathcal{X}_{0} \subset R_{\tau}(\Sigma)$. (In the following we will derive conditions ensuring existence and effective construction of $\mathcal{A}_{\mathcal{X}}$.) We now define system $S_{\lambda}(\Sigma)$ that approximates $S(\Sigma)$.

Definition 6: Suppose that $\Sigma$ in (3) is $\delta-\mathrm{FC}$ and for any accuracy $\lambda \in \mathbb{R}^{+}$define the system

$$
S_{\lambda}(\Sigma)=\left(X_{\lambda}, X_{0, \lambda}, U_{\lambda}, \longrightarrow, Y_{\lambda}, H_{\lambda}\right)
$$

where: $X_{\lambda}=\mathcal{A}_{\mathcal{X}}(\lambda) ; X_{0, \lambda}=\mathcal{A}_{\mathcal{X}_{0}}(\lambda) ; U_{\lambda}=U_{\tau}$; $x_{\lambda} \stackrel{u}{\longrightarrow} x_{\lambda}^{\prime}$ if $\left\|x_{\tau}\left(x_{\lambda}, u\right)-x_{\lambda}^{\prime}\right\|_{\infty} \leq \beta(\lambda, \tau)+\lambda$, where $\beta$ is the function appearing in (4); $Y_{\lambda}=\left[\mathbb{R}^{p}\right]_{\lambda}^{p} ; H_{\lambda}\left(x_{\lambda}\right)=$ $\left[z_{\lambda}\right]_{\lambda}^{p}$, with $z_{\lambda}=\left[\begin{array}{ll}I_{p} & 0\end{array}\right] x_{\lambda}(\tau)$, for all $x_{\lambda} \in X_{\lambda}$. In the sequel we use the symbol $x_{\lambda}$ to denote a state of $S_{\lambda}(\Sigma)$. If there exists a countable approximation $\mathcal{A}_{\mathcal{X}}$ of $R_{\tau}(\Sigma)$, system $S_{\lambda}(\Sigma)$ is countable. By definition of $X_{\lambda}$, system $S_{\lambda}(\Sigma)$ is metric; we use for $S_{\lambda}(\Sigma)$, the same metric $\mathbf{d}$ as the one used for $S(\Sigma)$. The following result establishes relations between $S(\Sigma)$ and $S_{\lambda}(\Sigma)$.

Theorem 2: Suppose that $\Sigma$ is $\delta$-FC and that there exists a countable approximation $\mathcal{A}_{\mathcal{X}}$ of $R_{\tau}(\Sigma)$. For any accuracy $\lambda \in \mathbb{R}^{+}$, the following statements hold:

(i) $S_{\lambda}(\Sigma) \preceq_{\lambda}^{s \text {,alt }} S(\Sigma)$ with strong A $\lambda$ A relation $\mathcal{R} \subseteq$ $X_{\lambda} \times X$ defined by $\left(x_{\lambda}, x\right) \in \mathcal{R} \Leftrightarrow\left\|x_{\tau}-x\right\|_{\infty} \leq \lambda$

(ii) $S(\Sigma) \preceq_{\lambda}^{s} S_{\lambda}(\Sigma)$ with strong $\lambda$ A relation $\mathcal{R}^{\prime} \subseteq X \times X_{\lambda}$ defined by $\mathcal{R}^{\prime}=\mathcal{R}^{-1}$

(iii) $\left\|H(x)-H_{\lambda}\left(x_{\lambda}\right)\right\| \leq \lambda$, for any $\left(x_{\lambda}, x\right) \in \mathcal{R}$.

Result above requires existence of a countable approximation $\mathcal{A}_{\mathcal{X}}$ of $R_{\tau}(\Sigma)$. The rest of this section is devoted to provide tools for the computation of $\mathcal{A}_{\mathcal{X}}$. We start by briefly recalling from [5] some results for approximating functional spaces, which are based on spline analysis, see e.g. [7]. Let us consider the space $\mathcal{Z} \subseteq C^{0}(I ; Z)$ with $Z \subseteq \mathbb{R}^{n}, I=[a, b]$,

\footnotetext{
${ }^{2}$ In fact the set $X$ of states of $S(\Sigma)$ is $\mathcal{X}$ and not $R_{\tau}(\Sigma)$. However, all states in $\mathcal{X} \backslash R_{\tau}(\Sigma)$ will be never reached and this is the reason why we will approximate $R_{\tau}(\Sigma)$ rather than $\mathcal{X}$.
}

$a, b \in \mathbb{R}$ and $a<b$. Given $N \in \mathbb{N}$ consider the following functions (see [7]):

$$
\begin{aligned}
& s_{0}(t)= \begin{cases}1-(t-a) / h, & t \in[a, a+h], \\
0, & \text { otherwise, }\end{cases} \\
& s_{i}(t)= \begin{cases}1-i+(t-a) / h, & t \in[a+(i-1) h, a+i h], \\
1+i-(t-a) / h, & t \in[a+i h, a+(i+1) h], \\
0, & \text { otherwise, }\end{cases} \\
& i=1,2, \ldots, N \text {; } \\
& s_{N+1}(t)= \begin{cases}1+(t-b) / h, & t \in[b-h, b], \\
0, & \text { otherwise, }\end{cases}
\end{aligned}
$$

where $h=(b-a) /(N+1)$. Functions $s_{i}$ called splines, are used to approximate $\mathcal{Z}$. The approximation scheme that we use is based on two steps:

- we first approximate function $z \in \mathcal{Z}$ by piecewiselinear function $z_{1}$, obtained as the linear combination of the $N+2$ splines $s_{i}$, centered at time $t=a+i h$ with amplitude $z(a+i h)$;

- we then approximate function $z_{1}$ by piecewise-linear function $z_{2}$, obtained as the linear combination of the $N+2$ splines $s_{i}$, centered at time $t=a+i h$ with amplitude $\tilde{z}_{i}$ in the lattice $[Z]_{\theta}^{n}$, which minimizes the distance from $z(a+i h)$, i.e. $\tilde{z}_{i}=\arg \min _{z \in[Z]_{\theta}^{n}} \| z-$ $z(a+i h) \|$.

Given any $N \in \mathbb{N}$ and $\theta, M \in \mathbb{R}^{+}$, let ${ }^{3}$ :

$$
\Lambda(N, \theta, M):=h^{2} M / 8+(N+2) \theta,
$$

with $h=(b-a) /(N+1)$. Function $\Lambda$ will be shown hereafter to be an upper bound for the error associated with the proposed approximation scheme. It is readily seen that for any $\lambda \in \mathbb{R}^{+}$and any $M \in \mathbb{R}^{+}$there always exist $N \in \mathbb{N}$ and $\theta \in \mathbb{R}^{+}$such that $\Lambda(N, \theta, M) \leq \lambda$. Let $N_{\lambda, M}$ and $\theta_{\lambda, M}$ be such that $\Lambda\left(N_{\lambda, M}, \theta_{\lambda, M}, M\right) \leq \lambda$. For any $\lambda \in \mathbb{R}^{+}$and $M \in \mathbb{R}^{+}$, define the operator $\psi_{\lambda, M}: \mathcal{Z} \rightarrow C^{0}([a, b] ; Z)$ that associates to any function $z \in \mathcal{Z}$ the following function

$$
\psi_{\lambda, M}(z)(t):=\sum_{i=0}^{N_{\lambda, M}+1} \tilde{z}_{i} s_{i}(t), \quad t \in[a, b],
$$

where $\tilde{z}_{i} \in[Z]_{\theta_{\lambda, M}}^{n}$ and $\left\|\tilde{z}_{i}-z(a+i h)\right\| \leq \theta_{\lambda, M}$, for any $i=0,1, \ldots, N_{\lambda, M}+1$. Note that operator $\psi_{\lambda, M}$ is not uniquely defined. For any given $M \in \mathbb{R}^{+}$and any given accuracy $\lambda \in \mathbb{R}^{+}$define:

$$
\mathcal{A}_{\mathcal{Z}, M}(\lambda):=\psi_{\lambda, M}(\mathcal{Z})
$$

Lemma 1: [5] Suppose that $\mathcal{Z} \subseteq P C^{2}(I ; Z)$ and there exists $M \in \mathbb{R}^{+}$such that $\left\|D^{2} z\right\|_{\infty} \leq M$ for any $z \in \mathcal{Z}$. Then $\mathcal{A}_{\mathcal{Z}, M}$ as defined in (13), is a countable approximation of $\mathcal{Z}$. Moreover, when $Z$ is bounded, $\mathcal{A}_{\mathcal{Z}, M}$ is a symbolic approximation of $\mathcal{Z}$.

Suppose that:

(A.1) $\Sigma$ in (3) is $\delta$-FC;

(A.2) set $X$ is bounded;

(A.3) functional $f$ is Fréchet differentiable in

\footnotetext{
${ }^{3}$ The real $M$ is a uniform bound on the norm of the second derivative of functions in $\mathcal{Z}$ and its role will become clear hereafter.
} 
$C^{0}\left([-\Delta, 0] ; \mathbb{R}^{n}\right) \times \mathbb{R}^{m}$

(A.4) The Fréchet differential $J(\phi, u)$ of $f$ is continuous and bounded on bounded subsets of $C^{0}\left([-\Delta, 0] ; \mathbb{R}^{n}\right) \times \mathbb{R}^{m}$. Under the assumptions above the following bounds are well defined:

$$
\begin{aligned}
B_{X} & =\sup _{x \in X}\|x\|, \quad B_{J}=\sup _{(\phi, u) \in C^{0}([-\Delta, 0] ; X) \times U}\|J(\phi, u)\|, \\
B_{U} & =\max _{u \in U}\|u\|, \quad M=\left(B_{X}+B_{U}\right) \kappa B_{J},
\end{aligned}
$$

where $\kappa$ is the Lipschitz constant of functional $f$ in the bounded set $C^{0}([-\Delta, 0] ; X) \times U$ and $\|J(\phi, u)\|$ denotes the norm of the operator $J(\phi, u): C^{0}\left([-\Delta, 0] ; \mathbb{R}^{n}\right) \times \mathbb{R}^{m} \rightarrow \mathbb{R}^{n}$. The following result holds.

Theorem 3: Suppose that the time-delay system $\Sigma$ is $\delta$-FC and that it satisfies Assumptions (A.1-5). For any quantization parameter $\lambda \in \mathbb{R}^{+}$, pick $\theta \in \mathbb{R}^{+}$and $N \in \mathbb{N}$ satisfying the following inequality

$$
\Lambda(N, \theta, M) \leq \lambda,
$$

with $\Lambda$ as in (11) and $M$ as in (14). Then, the following statements hold:

(i) $S_{N, \theta}(\Sigma)$ is symbolic;

(ii) $S_{N, \theta}(\Sigma) \preceq_{\lambda}^{s, \text { alt }} S(\Sigma)$ with strong $\mathrm{A} \lambda$ A relation $\mathcal{R} \subseteq$ $X_{\lambda} \times X$ defined by $\left(x_{\lambda}, x\right) \in \mathcal{R} \Leftrightarrow\left\|x_{\tau}-x\right\|_{\infty} \leq$ $\Lambda(N, \theta, M)$

(iii) $S(\Sigma) \preceq_{\lambda}^{s} S_{N, \theta}(\Sigma)$ with strong $\lambda$ A relation $\mathcal{R}^{\prime} \subseteq X \times$ $X_{\lambda}$ defined by $\mathcal{R}^{\prime}=\mathcal{R}^{-1}$

(iv) $\left\|H(x)-H_{\lambda}\left(x_{\lambda}\right)\right\| \leq \Lambda(N, \theta, M)$, for any $\left(x_{\lambda}, x\right) \in \mathcal{R}$.

\section{Vi. Application to the Artificial Pancreas}

In this section, the symbolic approach presented in the previous sections is applied to the Artificial Pancreas framework. The chosen model is a time-delay system that has been shown to be effective and reliable both (i) in explaining the glucose-insulin dynamics and assessing individual insulin resistance [16], [15], and (ii) in designing modelbased glucose control therapies for T2DM patients, with a special focus on observer-based closed-loop control laws, where insulin (unaffordable for real-time measurements) is estimated by means of state observers [17], [18], [25], [26], [27]. The dynamic equations at time $t \geq 0$ are given by:

$$
\left\{\begin{array}{l}
\dot{G}(t)=-K_{x g i} G(t) I(t)+\frac{T_{g h}}{V_{G}}, \\
\dot{I}(t)=-K_{x i} I(t)+\frac{T_{i G \max }}{V_{I}} h(G(t-\Delta))+\frac{u(t)}{V_{I}},
\end{array}\right.
$$

starting from initial conditions $G(t)=G_{0}(t), I(t)=I_{0}(t)$, $t \in[-\Delta, 0]$, where $G(t),[m M]$, and $I(t),[p M]$, denote the plasma glucose and insulin concentrations at time $t . h(G)$ is a nonlinear Hill function $h(G)=\frac{\left(G / G^{*}\right)^{\gamma}}{1+\left(G / G^{*}\right)^{\gamma}}$ representing the endogenous pancreatic IDR, $u(t)$ [pmol $/ \mathrm{kgBW} / \mathrm{min}$ ] is the control input in terms of exogenous intra-venous IDR at time $t$ (see [16], [15] for any further details). The system described above matches the form (1) with $r=0$, where we consider the continuous output $y(t)=G(t)$ available from measurements. This model possesses some nice formal properties, among which we recall that it is a positive system [28] with unique locally asymptotically stable equilibrium point at the basal state $\left(G_{b}, I_{b}\right)$, as well as uniform lower and upper bounds on its continuous evolution (see [15] for further details). The parameters of the model are taken from Table 1 in [18], with equilibrium given by $G_{b}=8.45[\mathrm{mM}]$ and $I_{b}=47.85[p M]$. We set $G_{\max }=20, I_{\max }=1000$, in agreement with the uniform bounds computable as in [15] and with some preliminary simulations, which delimit the working region of the system, and we assume a bounded input with $u(t) \leq u_{\max }=100[\mathrm{pmol} / \mathrm{kgBW} / \mathrm{min}]$ for all times $t$. For ease of computation, we rescale state and input variables by considering $G(t) / G_{\max }, I(t) / I_{\max }$ and $u(t) / u_{\max }$, instead of $G(t), I(t)$ and $u(t)$, leading to the constraining sets $X=[0,1[\times[0,1[, U=0.02 \mathbb{Z} \cap$ $[0,1$. We consider a $\delta$-FC Lyapunov-Krasovskii functional $V(\phi, \psi)=\left(\phi_{1}(0)-\psi_{1}(0)\right)^{2}+\gamma_{1}\left(\phi_{2}(0)-\psi_{2}(0)\right)^{2}+$ $\gamma_{2} \int_{-\Delta}^{0}\left(\phi_{1}(t)-\psi_{1}(t)\right)^{2} e^{t} d t$, as in Definition 2, with $\gamma_{1}=$ $\gamma_{2}=1$, with $\alpha_{1}(x)=\min \left\{1, \gamma_{1}\right\}, \alpha_{2}=\max \left\{1, \gamma_{1}\right\}+\gamma_{2} \Delta$, and $p=1$ in (6). Hence, as a consequence of Theorem 1 , the system is $\delta-\mathrm{FC}$, satisfying property (A.1) in Section V. Property (A.2) is guaranteed by the uniform boundedness $G(t) \leq G_{\max }$ and $I(t) \leq I_{\max }$. Properties (A.3), (A.4), (A.5) are satisfied for $B_{X}=1, B_{J}=1.67, B_{U}=1, M=0.13$, $\kappa=0.038$, where the sampling time $\tau=15$ [min] is chosen, which is a multiple of a common sampling interval (5 minutes) characterizing many off-the-shelf Continuous Glucose Monitoring (CGM) devices [29]. We set $\lambda=0.1$ and we choose $N=8$ and $\theta=0.005$ satisfying (15), resulting in a symbolic model with 826,281 states. We now use the computed symbolic model to solve a typical glucose control problem. We consider a population of 20 diabetic (virtual) patients, each modeled by the equations in (16) and whose parameters are uniformly randomly perturbed up to $10 \%$ with respect to the nominal values in [18] and considered in the construction of the symbolic model. According to the modified parameters, each patient has a different equilibrium with respect to $\left(G_{b}, I_{b}\right)$, and we assume to start each simulation from the corresponding equilibrium. Since the basic step in glucose control is to regulate the level of fasting glycemia by means of exogeneous insulin infusions, we consider a time horizon of 5 hours, neglecting the effect of meals. Since the glycemic behavior is highly dependent on individual features (even in perfectly healthy people), it is common medical practice to attempt to drive the glycemia into an interval $\left[G_{L B}, G_{U B}\right]$, rather than tracking an exact set point. We choose $G_{L B}=3.9$ and $G_{U B}=6$, where the chosen values are considered as alerts for severe hypoglycemia (occurring for $G(t)<3$ ) and for a pre-diabetic condition (the so-called Impaired Fasting Glycemia, IFG), respectively. For solving this control problem, we formulated a 'reach and stay' specification that has been solved by using supervisory control type algorithms [21], [22] on the symbolic model we computed. According to the obtained simulation results, all the controlled patients readily decrease their glycemia (due to the control action) below the hyperlycemic threshold, and no (severe) hypoglycemiae are detected for the considered virtual population at any time, indicating that the controller 

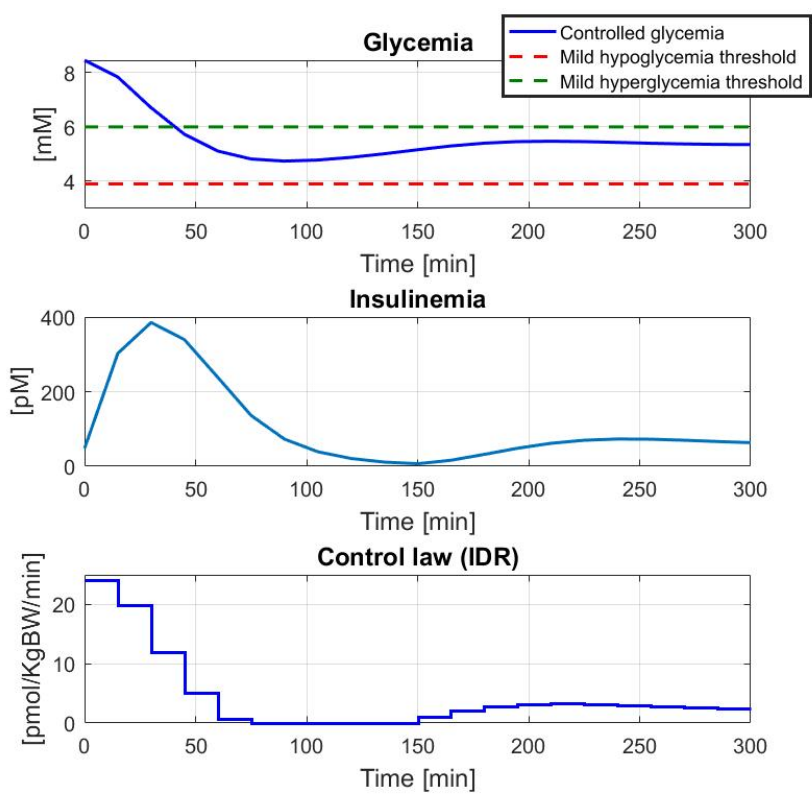

Fig. 1. Glycemic behavior of a virtual patient controlled by means of a feedback law extracted from the symbolic model.

is able to avoid dangerous conditions robustly with respect to (limited) parameter variations. The plot in Fig. 1 illustrates a random realization of the controlled system for one of the patients, in terms of state variables and control input, where we observe that the specification is met.

\section{CONCLUSION}

In this paper we proposed symbolic models that can be effectively constructed and that approximate $\delta$-FC timedelay systems with quantized measurements of the output, in the sense of approximate alternating simulation. The results were then applied to a well-known model of the glucoseinsulin system to solve a glucose control problem in presence of parameter uncertainties.

Acknowledgement 1: The authors would like to thank Andrea De Gaetano and Simona Panunzi for fruitful discussions on artificial pancreas.

\section{REFERENCES}

[1] P. Tabuada, Verification and Control of Hybrid Systems: A Symbolic Approach. Springer, 2009.

[2] C. Belta, B. Yordanov, and E. Aydin Gol, Formal Methods for Discrete-Time Dynamical Systems. Springer, 2017.

[3] M.D. Di Benedetto and G. Pola, "Networked embedded control systems: from modelling to implementation," in Proceedings of the third Conference Hybrid Autonomous Systems, ser. Electronic Proceedings in Theoretical Computer Science (EPTCS) 124, P. G. Bortolussi L., Bujorianu M.L., Ed., 2013, pp. 9-13.

[4] M. Zamani, M. Mazo, G. Pola, and P. Tabuada, "Symbolic models for nonlinear control systems without stability assumptions," IEEE Trans. Automatic Control, vol. 57, no. 7, pp. 1804-1809, 2012.

[5] G. Pola, P. Pepe, M.D. Di Benedetto, and P. Tabuada, "Symbolic models for nonlinear time-delay systems using approximate bisimulations," Systems and Control Letters, vol. 59, pp. 365-373, 2010.
[6] G. Pola, P. Pepe, and M.D. Di Benedetto, "Symbolic models for timevarying time-delay systems via alternating approximate bisimulation," Int. J. Robust Nonlin. Control, vol. 25, pp. 2328-2347, 2015.

[7] M. H. Schultz, Spline Analysis. Prentice Hall, 1973.

[8] G. Pola and M.D. Di Benedetto, "Approximate supervisory control of nonlinear systems with outputs," in 56th IEEE Conference on Decision and Control, Melbourne, Australia, December 2017, pp. 2991-2996.

[9] C. Cobelli, E. Renard, and B. Kovatchev, "Artificial pancreas: past, present, future," Diabetes, vol. 60, pp. 2672-2682, 2011.

[10] F. Doyle III, L. Huyett, J. Lee, H. Zisser, and E. Dassau, "Closedloop artificial pancreas systems: Engineering the algorithms," Diabetes Care, vol. 37, pp. 1191-1197, 2014

[11] R. Hovorka, "Closed-loop insulin delivery: From bench to clinical practice," Nature Rev. Endocrinol., vol. 7, no. 7, pp. 385-395, 2011.

[12] B. Kovatchev, W. Tamborlane, W. Cefalu, and C. Cobelli, "The artificial pancreas in 2016: A digital treatment ecosystem for diabetes," Diabetes Care, vol. 39, pp. 1123-1126, 2016.

[13] K. Zarkogianni, E. Litsa, K. Mitsis, P. Wu, C. Kaddi, C. Cheng, M. Wang, and K. Nikita, "A review of emerging technologies for the management of diabetes mellitus," IEEE Trans. Biomed. Eng., vol. 62, no. 12, pp. 2735-2749, 2015.

[14] L. Kovacs, "A robust fixed point transformation-based approach for type 1 diabetes control," Nonlinear dynamics, vol. 89, no. 4, pp. 24812493, 2017.

[15] P. Palumbo, S. Panunzi, and A. De Gaetano, "Qualitative behavior of a family of delay-differential models of the glucose-insulin system," Discrete and Continuous Dynamical Systems Series B, vol. 7, no. 2, pp. 399-424, 2007.

[16] S. Panunzi, P. Palumbo, and A. De Gaetano, "A discrete single delay model for the intra-venous glucose tolerance test," Theoretical Biology and Medical Modelling, vol. 4, no. 1, p. 35, 2007.

[17] P. Palumbo, P. Pepe, S. Panunzi, and A. De Gaetano, "Time-delay model-based control of the glucose-insulin system, by means of a state observer," European Journal of Control, vol. 6, pp. 591-606, 2012.

[18] P. Palumbo, G. Pizzichelli, S. Panunzi, P. Pepe, and A. De Gaetano, "Model based control of plasma glycemia: Tests on populations of virtual patients," Math. Biosciences, vol. 257, pp. 2-10, 2014.

[19] A. Makroglou, J. Li, and Y. Kuang, "Mathematical models and software tools for the glucose-insulin regulatory system and diabetes: an overview," Applied Numerical Mathematics, vol. 56, pp. 559-573, 2006.

[20] P. Palumbo, S. Ditlevsen, A. Bertuzzi, and A. De Gaetano, "Mathematical modeling of the glucoseinsulin system: A review," Math. Biosci., vol. 244, pp. 69-81, 2013.

[21] C. Cassandras and S. Lafortune, Introduction to discrete event systems. Boston, MA: Kluwer Academic Publishers, 1999.

[22] G. Pola, P. Pepe, and M. D. Di Benedetto, "Decentralized approximate supervisory control of networks of nonlinear control systems," IEEE Trans. Automatic Control, vol. 63, pp. 2803-2817, 2018.

[23] G. Pola and M.D. Di Benedetto, "Approximate supervisory control of nonlinear systems with outputs," in 56th IEEE Conference on Decision and Control, Melbourne, Australia, December 2017, pp. 2991-2996.

[24] A. Borri, G. Pola, and M. D. Di Benedetto, "Design of symbolic controllers for networked control systems," IEEE Trans. Automatic Control,, vol. 64, pp. 1034-1046, 2019.

[25] A. Borri, F. Cacace, A. De Gaetano, A. Germani, C. Manes, P. Palumbo, S. Panunzi, and P. Pepe, "Luenberger-like observers for nonlinear time-delay systems with application to the artificial pancreas: The attainment of good performance," IEEE Control Systems Magazine, vol. 37, no. 4, pp. 33-49, 2017.

[26] J. Bondia, S. Romero-Vivo, B. Ricarte, and J. Diez, "Insulin estimation and prediction: A review of the estimation and prediction of subcutaneous insulin pharmacokinetics in closed-loop glucose control," IEEE Control Systems Magazine, vol. 38, no. 1, pp. 47-66, 2018.

[27] A. Borri, P. Palumbo, C. Manes, S. Panunzi, and A. De Gaetano, "Sampled-data observer-based glucose control for the artificial pancreas," Acta Polytech. Hungarica, vol. 14, no. 1, pp. 79-94, 2017.

[28] L. Farina and S. Rinaldi, Positive linear systems: theory and applications. John Wiley \& Sons, 2011, vol. 50.

[29] D. Keenan, Barry, Mastrototaro, J. John, Voskanyan, Gayane, Steil, and M. Garry, "Delays in minimally invasive continuous glucose monitoring devices: a review of current technology," Journal of diabetes science and technology, vol. 3, no. 5, pp. 1207-1214, 2009. 